SLAC-TN-12-028

\title{
Longitudinal Bunch Pattern Measurements through Single Photon Counting at SPEAR3
}

\author{
Hongyi (Jack) Wang \\ Office of Science, Science Undergraduate Laboratory Internship (SULI) Program \\ UCSD Electrical Engineering Class of 2013 \\ SLAC National Accelerator Laboratory \\ Stanford, CA \\ $08 / 03 / 2011$
}

Prepared in partial fulfillment of the requirements of the Office of Science, Department of Energy's Science undergraduate Laboratory Internship under the direction of Jeff Corbett at SSRL, SLAC National Accelerator Laboratory.

Participant:

Signature

Research Advisor:

Signature 


\section{Table of Contents:}

$\begin{array}{ll}\text { Abstract } & 2\end{array}$

$\begin{array}{ll}\text { Introduction } & 3\end{array}$

$\begin{array}{ll}\text { Materials and Methods } & 7\end{array}$

$\begin{array}{ll}\text { Results } & 11\end{array}$

$\begin{array}{ll}\text { Conclusion } & 14\end{array}$

$\begin{array}{ll}\text { Acknowledgement } & 15\end{array}$

$\begin{array}{ll}\text { Reference } & 16\end{array}$ 
Longitudinal Bunch Pattern Measurements through Single Photon Counting at SPEAR3. HONGYI WANG (University of California, San Diego, La Jolla, CA 92093). Jeff Corbett (SSRL Operations, SLAC National Laboratory, Menlo Park, CA 94025)

\section{Abstract}

The SSRL is a synchrotron light source that provides x-rays for experimental use. As electrons are bent in the storage ring, they emit electromagnetic radiation. There are 372 different buckets which electrons can be loaded into. Different filling patterns produce different types of $x$-rays. What is the bunch pattern at a given time? Which filling pattern is better? Are there any flaws to the current injection system? These questions can be answered with this single photon counting experiment.

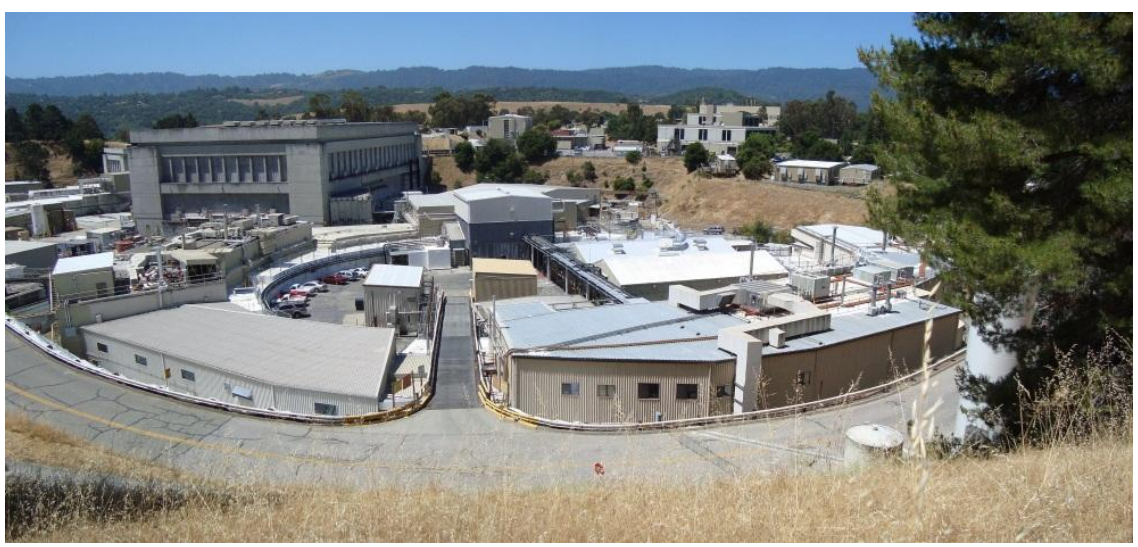




\section{Introduction}

The Stanford Synchrotron Radiation Lightsource (SSRL) is a division of SLAC National Accelerator Laboratory. SSRL provides to users electromagnetic radiation in $\mathrm{x}$-ray, ultraviolet, visible, and infrared spectrums for a variety of experiments ranging from basic materials science to advanced protein crystallography and biological research. The various portions of the electromagnetic spectrum are delivered to 14 different beam-lines by means of mirrors, collimators, and aggressively-cooled grating and crystal monochromaters. The electromagnetic radiation is given off as synchrotron radiation by electron bunches stored in the SPEAR3 ring. The electron bunches can be loaded into 372 different buckets, which are radio-frequency power potential wells in the storage ring. Each electron bunch produces synchrotron radiation as they are radially accelerated in bend magnets or more advanced wiggler and undulator magnets.

The circumference of the SPEAR3 storage ring is $234.12 \mathrm{~m}$. The electron bunches therefore revolve with a turn time of $780 \mathrm{~ns}$, corresponding to a $1.28 \mathrm{MHz}$ repetition rate. When all 372 buckets contain electrons, experimenters at the photon beam lines see a photon-beam pulse repetition rate up to $476 \mathrm{MHz}$. With a maximum of $500 \mathrm{~mA}$ total of circulating electron beam current in SPEAR3, the total synchrotron radiation (SR) power ranges up to $450 \mathrm{~kW}$; however, after filtering and collimation, a typical beam line only receives a few $\mathrm{mW}$ of photon beam power. For most applications, SR Users are interested in average beam power and not the pulse-by-pulse time structure generated by the individual bunches separated by 2.1 ns. Nevertheless, the 372 different buckets can be filled with different electron 'bunch' patterns.

Dark-space or 'gaps' are normally left between trains of electron bunches, to prevent the trapping and accumulation of positively-charged ions in the SPEAR3 vacuum chamber. As electrons travel through the storage ring, there are some positively charged particles flowing around the tube, 
despite vacuuming efforts. If the buckets are filled without gaps, the continuous electron beam will attract positively charge particles inside the vacuum chamber towards the beam. As a result, the positively charged particle will be attracted towards the electrons and continuously deflect the electron beam. The trapped ions can lead to electron beam instability and particle loss, due to elastic and inelastic collisions. For this reason, some buckets are left unfilled, to give positively charged particles time to escape the electric field from the beam and thus reduce deflection. A second reason for leaving 'gaps' in the electron bunch pattern is to produce different types of time-dependent radiation for various experimental needs. Certain measurements prefer single isolated photon pulses to image timedependent sample dynamics. Often a laser is used to 'pump' the sample while the x-ray beam is used to 'probe' the response. Since the fast-gating of the detectors requires up to several hundred nanoseconds, the probe pulse must be isolated from the rest of the bunch pattern. Of significance to this study is the fact that the probe pulse must be very well isolated with charge in adjacent buckets down by a factor of $10^{-5}$ or less. Some fill patterns are better than others, due to reasons that will be discussed later.

The fill pattern in the SPEAR3 ring is established by a graphical-interface control system that communicates with a complex timing system from the SSRL control room. From the control room, operators inject beam into the individual SPEAR3 buckets, and can monitor the electron bunch pattern as detected from a capacitive pick-up electrode with relative ease; however, this does not have the dynamic range required for isolated-bunch timing experiments. Often it is difficult to detect timing flaws with the current injection system, which can be attributed to frequency drift, electronic jitter or temperature-dependent phase-errors that accumulate in the long-haul timing cables extending between the SPEAR3 control room and the injector. All of these effects can lead to inadvertent injection of charge in buckets adjacent to the intended target bucket. These are the causes of some of the questions investigated with single photon counting experiments carried out in the SSRL synchrotron light monitor diagnostic room, called the light-shack. 
By utilizing a single photon counting technique, the electron bunch pattern in the SPEAR3 storage ring was able to be mapped out. From the user side, it was determined which buckets were filled, which were left empty, and in relative terms how many electrons were in each bucket. This information can be used to confirm with the control room to ensure good beam delivery to each beamline. Although there are other ways to measure the bunch pattern, as previously mentioned, singlephoton yields the highest dynamic range. For other applications, such as laser-SR beam cross-correlation to measure individual bunch length, photon production from the cross-correlation experiment is so slow that single-photon counting is the only way to measure the result. Many other single-photon counting applications occur on the SSRL beam lines and throughout both applied physics and low-light intensity measurements, such as those found in astronomy.

\section{Single-Photon Counting Technique:}

The technique of mapping the location and relative radiation power of each electron bunch is known as Single Photon Counting. Basically, this requires counting the number of photons hitting the detector from each electron bunch; as the name of the technique suggests, the number of photons hitting are in the single photon range. Therefore, the synchrotron radiation light must be of such low intensity that only one photon strikes the detector in a single counting interval. To do this, a photomultiplier tube (PMT) is used as the detector. A photomultiplier tube consists of a photocathode, electron multipliers, and an anode. When a photon hits the photocathode, electrons are emitted under the photoelectric effect. The number of electrons is then multiplied through cascade electron emissions from the dynodes, or electron multipliers. These electrons are collected by the anode as a voltage pulse output. The output of the PMT is an analog TTL signal for each photon detected, and a fast digitizer is needed to translate and store the output signal in the desired data format. With single photon counting, each pulse in the output signal represents one photon hitting the detector. Since each photon is 
generated by an orbiting electron bunch in the ring, the timing of the pulses can be tracked, to extrapolate the position of each electron bunch in the storage ring relative to each other.

At nominal electron beam currents of several hundred milliamps in SPEAR3, the photon entering the diagnostic room spans a range of approximately 420-1000 nm, and has a beam power of about 100 uW, which contains way too many photons than we need. Band-pass filters and wide-band neutraldensity filters, or "sunglasses", are therefore used to control the amount of power incident on the single-photon counter. Filtering the synchrotron radiation to the single photon range is important, because at high light levels, multiple photons will hit the detector at once. This runs the risk of overloading the detector, as too many electrons may be emitted at one time. Also, if photons hit the detector too frequently, the output pulses will overlap with each other (Figure 1). As a result, there will be a waveform of the train of photons, but no information about each individual photon arrival. As we are interested in studying the individual buckets in the storage ring, as well as the overall distribution, photon counting will give us more accurate information at low light level.

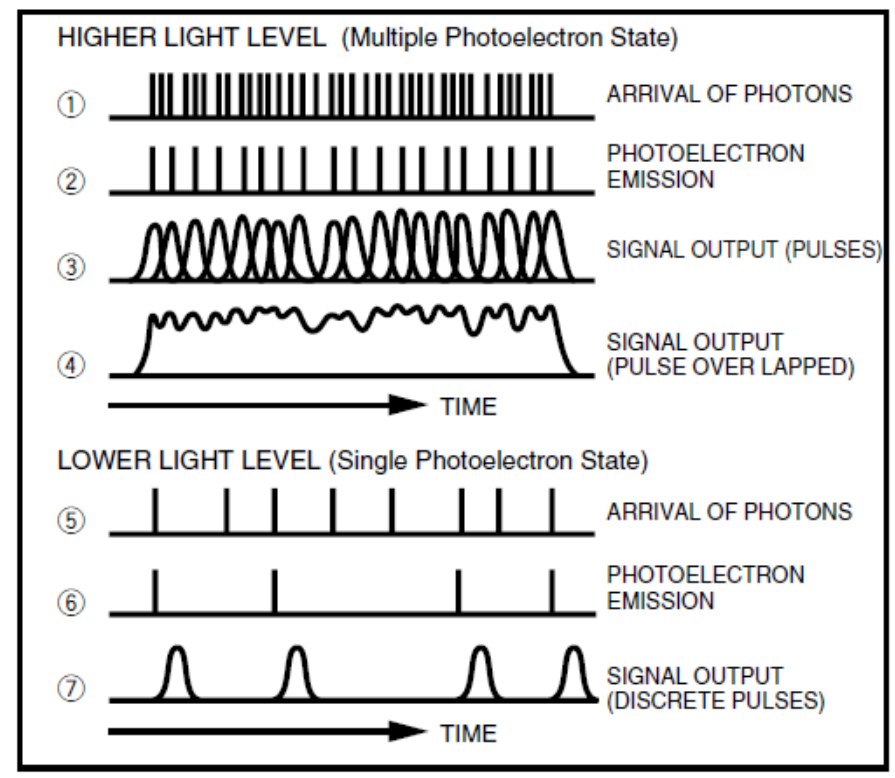

1 Figure: Output Pulses from Photomultiplier Tube at Different Light Levels At high light level, signal output pulses overlap, and single photon arrivals cannot be resolved. At low light levels, signal outputs are discrete pulses, and single photon arrival can be resolved. 
With single photon counting, the beam is filtered down to the 'single photon' range to avoid pulse overlaps. This also means that only a few photons hit the detector in one turn, and only limited information about the electron bunches can be gathered in one rotation in the ring. For typical singlephoton counting applications, one wants to detect only about 1 photon in every 10 counting intervals, to avoid overload. To solve this problem, a time-correlated integration technique was used. Since only the relative position of electron bunches was of interest, the signal could be integrated from the detector, as electron bunches revolve for multiple turns. Although only a few photons are let through each pass over many turns in the ring, each photon has an equal probability of hitting the detector. While only several pulses are collected in one turn, over millions of turns the full electron bunch profile can be mapped out. Each turn takes 780 ns, so integrating over millions of turns would still allow a reasonable short sampling time.

\section{Materials and Methods}

The single-photon counting experiments were conducted in the SSRL Light-Shack. The LightShack is a special diagnostic beam-line that only collects radiation in the visible, near IR spectrum (420nm-1000nm). For this experiment, harmful radiation in the $\mathrm{x}$-ray range is not needed, since visible spectrum photons convey the same message about the electron bunch pattern. Although x-ray radiation is filtered out, other properties of the radiation from this diagnostic beam-line is the same as other experimental beam-lines. Therefore, the light-shack is an ideal location in which to conduct this experiment. 
When the light comes out of the beam-line, it is guided onto an optical bench. Through a series of focusing mirrors and focusing lenses, the beam is then directed into a light-tight box where the detector sits. In order to filter the incident photon beam down to single-photon counting regime, a combination of neutral density filters and a $10 \mathrm{~nm}$ band-pass filter centered at $550 \mathrm{~nm}$ were used. Through measurements with a Newport Power Meter, the average power of the beam was measured (Table 1). An estimate of number of photons per turn was calculated using the equation below:

$$
\text { Beam Power }=\frac{N \times h \times c}{T \times \lambda}
$$

( $\mathrm{N}$ represents the number of photons, and is the only unknown variable)

\begin{tabular}{|c|c|c|}
\hline $\begin{array}{c}\text { Neutral Density } \\
\text { Filter Level }\end{array}$ & Measured Beam & \\
\hline 0 & 206 & Number Of Photons in Beam Per Turn (780 ns) \\
\hline 1 & 20.4 & 993123456.8 \\
\hline 2 & 2.58 & 12438148.15 \\
\hline 3 & 0.458 & 2208012.346 \\
\hline 4 & 0.0712 & 343254.321 \\
\hline 5 & 0.01 & 48209.87654 \\
\hline
\end{tabular}

Table 1: Measured beam power from power-meter and corresponding number of photons at each filter level. No band-pass filter used.

SSRL Running at $350 \mathrm{~mA}$.

Extrapolating from the data in Table 1, a level 8 neutral density filter in series with a $10 \mathrm{~nm}$ band-pass filter only lets through 1 photon out of every 10 turns. Ideally, the neutral-density filters should correspond to beam power reduction in powers of 10 . Our results roughly follow the pattern, 
some deviate may be caused by noise and sensitivity of detector. ND8 therefore reduces beam power by a factor of $10^{7}$. This filtering achieves the desired single-photon range, but slight adjustments are made in accordance with sampling time: fewer photons hitting means a longer sampling time is necessary to produce the same result. The detector was sealed light-tight to block out ambient room light noise, and experiments were run with room lights turned off.
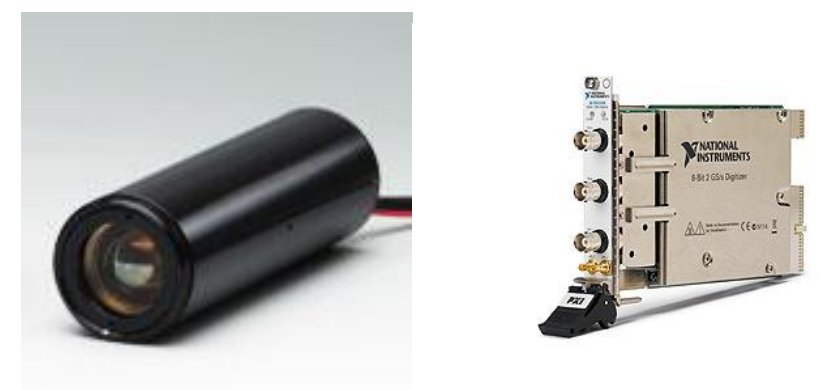

Figure 2: (Left) Hamamatsu Photonics H-7360 Photomultiplier Tube. (Right) National Instruments 5154 2Gs/s Digitizer

The detector chosen was a Hamamatsu Photonics H-7360 Counting Head. This detector contains a photomultiplier tube that is sensitive to single photons. The detector can be biased with a DC voltage between 3.4 $\mathrm{V}$ and $5 \mathrm{~V}$. When a photon hit the detector, a TTL pulse was generated from the anode. This TTL pulse was then sent to a National Instrument 5154 2GSample/s Digitizer for analysis. The digitizer was triggered from the constant 1.28 MHz SSRL ring-clock, a TTL pulse provided from the control room synchronous with the revolution time of the individual bunches. For each ring-clock trigger, the TTL pulses were saved as a 'time-of-flight' record for the single-photon arrival times. A Ortec Counting Card was intended to be used originally. It is also a digitizer and produces very high resolution histograms. The Ortec Card was tested with DG535 generators, which generated simulated TTL pulses, and the setup was ready to take actual data. However, the counting card was taken to a different beam-line due to a conflict in experiments, and the National Instruments Digitizer was used instead. 
After preparing the detector, software was developed to communicate with the detector output and extract the desired data from the analog TTL signals. Using a Lab-View Module that makes time histograms, we modified the program to work for our specific experiment. With the help with a experienced LabView programmer, we connected all terminals of the block diagram and correct input to parameters were given. Our final program keeps track of all TTL pulses from the detector and makes a time histogram evaluated over the rotating period of the ring, which is $780 \mathrm{~ns}$. Output is stored in a text file. Since each TTL pulse indicates the presence of an electron bunch in the storage ring, a histogram that keeps track of all TTL pulse generates a full longitudinal electron bunch pattern over the course of time. Figure 3 is a screen shot of the user interface of the program.

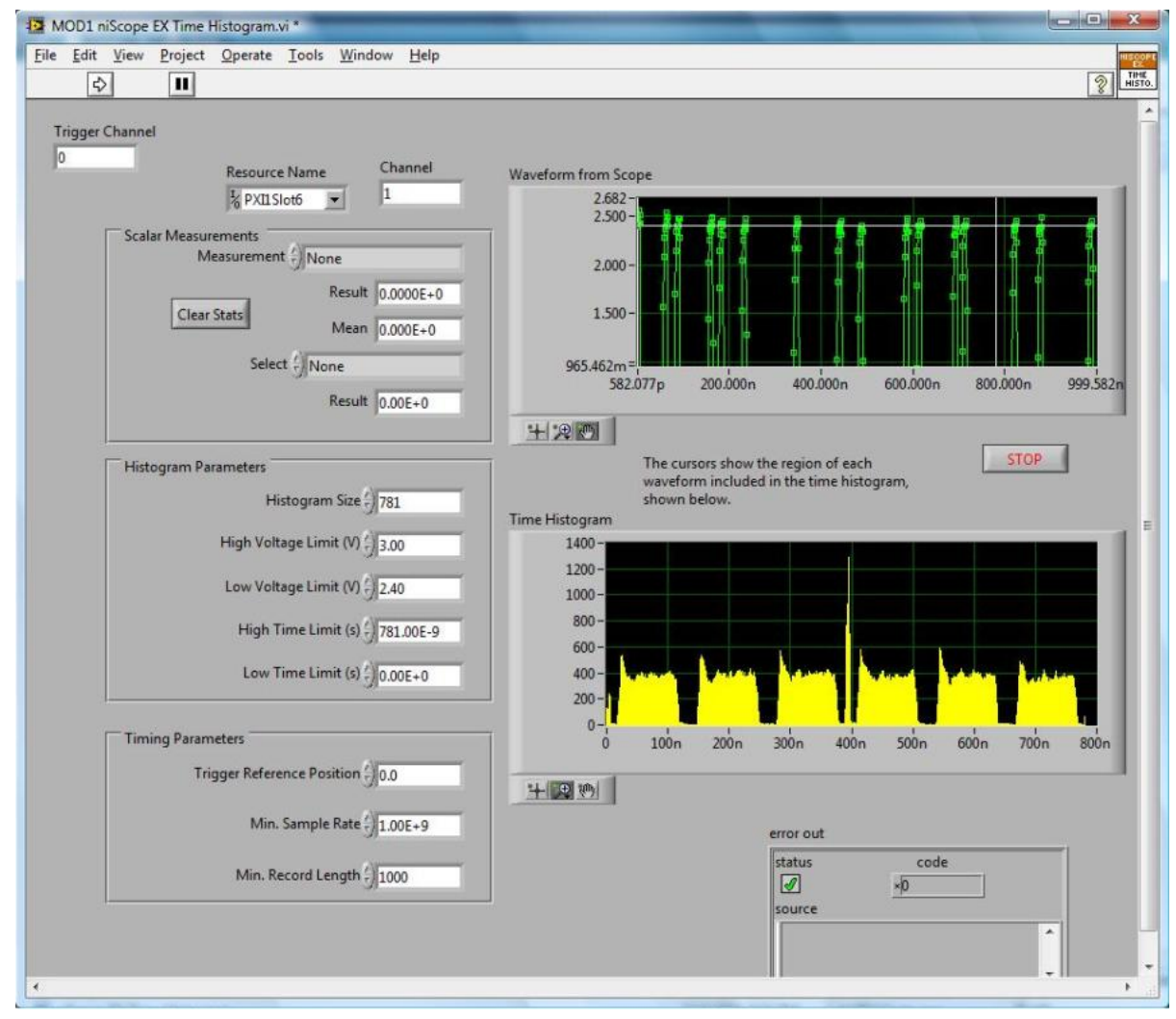

Figure 3: User Interface of Lab View Program

The software contains a Scope function (top right display) that keeps track of TTL pulses after a single trigger. The time-correlated Integration is done in the histogram (bottom right display) that integrates the data from Scope. 


\section{Program Parameters:}

\begin{tabular}{|c|c|c|}
\hline Parameter Name: & Description: & What was used in experiment: \\
\hline Histogram Size & $\begin{array}{l}\text { number of bins contained in the } \\
\text { histogram, histogram resolution. }\end{array}$ & 781 ( 2 bins $=1$ bucket $)$ \\
\hline $\begin{array}{l}\text { High Voltage Limit/Low Voltage } \\
\text { Limit }\end{array}$ & $\begin{array}{c}\text { This defines the range which the } \\
\text { program samples data and includes in } \\
\text { the histogram. }\end{array}$ & $\begin{array}{l}\text { 2.4V-3.0V (signal from detector falls } \\
\text { within this range) }\end{array}$ \\
\hline High Time Limit/Low Time Limit & This defines the $x$-axis of the histogram & $\begin{array}{l}\text { Ons-781ns(represents the turn time of } \\
\text { SSRL) }\end{array}$ \\
\hline Trigger Reference Position & $\begin{array}{l}\text { defines the position of the trigger } \\
\text { relative to data. At } 0 \text {, all data collected } \\
\text { are after the trigger. At } 100 \text {, all data } \\
\text { collected are prior to trigger. }\end{array}$ & 0 (all data collected after trigger) \\
\hline Min Sample Rate & $\begin{array}{l}\text { This is the actual sample rate. (1 Ghz } \\
\text { maximum) }\end{array}$ & $1 \mathrm{Ghz}$ (produces the highest resolution) \\
\hline Min Record Length & $\begin{array}{l}\text { This defines the time length which the } \\
\text { detector looks for data after a trigger. It } \\
\text { specifies the number of sample points } \\
\text { the scope looks for. It interacts with } \\
\text { sample rate to define the time length } \\
\text { which the detector looks for data after a } \\
\text { trigger (Example. At } 1 \mathrm{Ghz}, 700 \text { for } \\
\text { record length = samples for } 700 \mathrm{~ns} \text { after } \\
\text { trigger). }\end{array}$ & $\begin{array}{l}781 \text { (samples } 781 \text { data points at } 1 \mathrm{Ghz} \\
\text { after trigger, which is } 781 \mathrm{~ns} \text { ) }\end{array}$ \\
\hline
\end{tabular}

Table 2: Lab View Program Parameters

This table contains an description of all parameters associated with our software, as well as the values we used for our experiment.

\section{Results}

Using our experimental setup, we were able to get very good data. A few different bunch patterns were detected and analyzed.

The Following results were taken during normal SSRL operations, when the pattern was run for user experiments. 


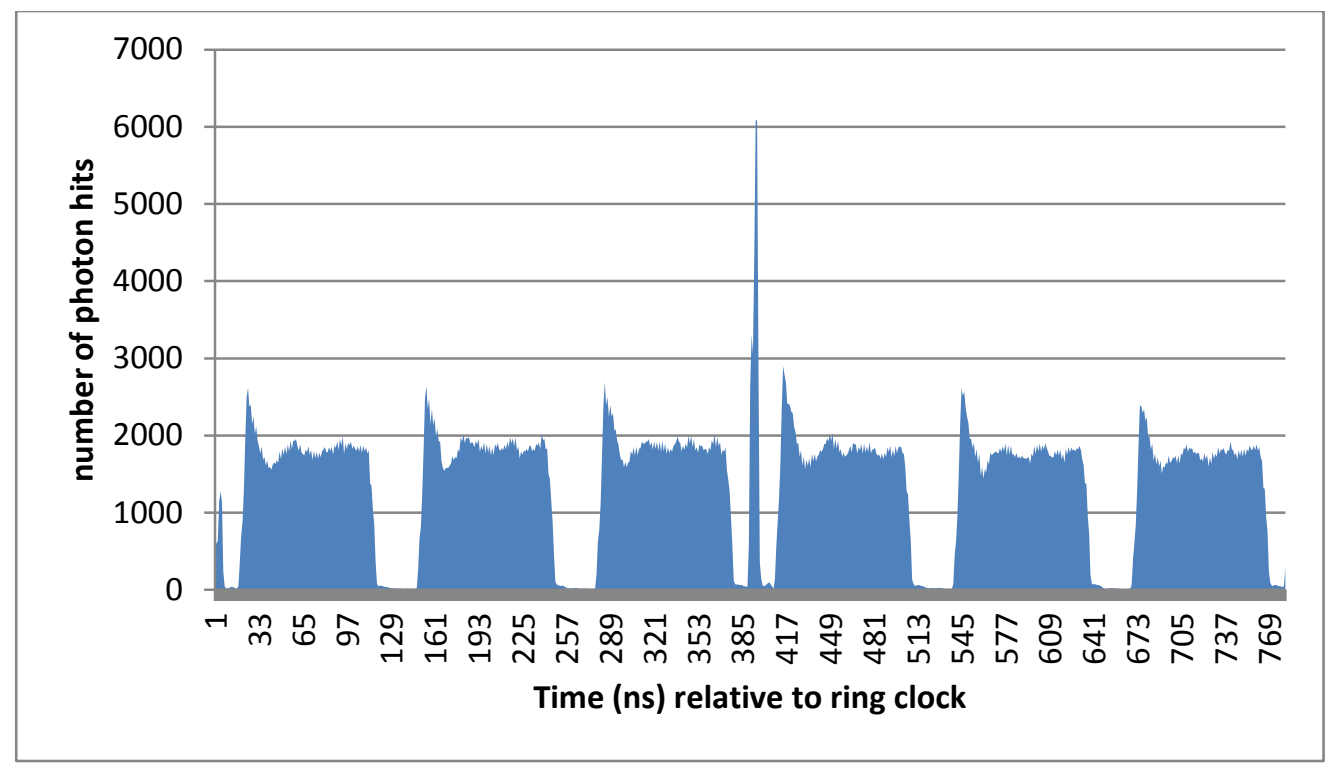

Figure 5: 6 Electron Bunch Train with 1 Mondo Bunch 45 bunches per train with 17 bunch gap

5 min Sampling Time

Taken at 4:09pm 07/22/2011

This pattern is what the users see from their beam-line for their experiments. The bunch trains are used as a continuous radiation source. The Mundo bunch is an isolated bunch with high current used for pump-probe experiments.

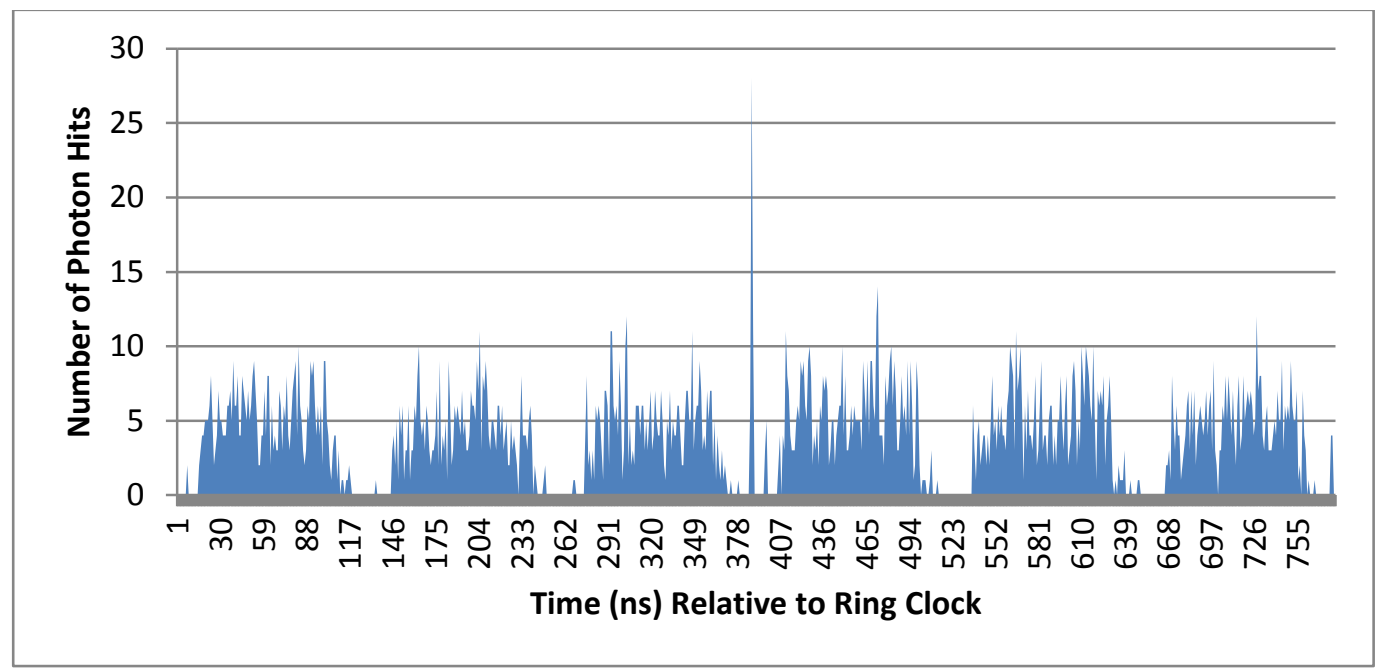

Figure 6: 6 Electron Bunch Train with 1 Mondo Bunch

45 bunches per train with 17 bunch gap

5 sec Sampling Time

Taken at 5:40pm 7/20/2011

Results are fairly noisy due to short sampling time. 
The following results were taken during accelerator physics beam time. These bunch patterns are used for SSRL facility diagnostics.

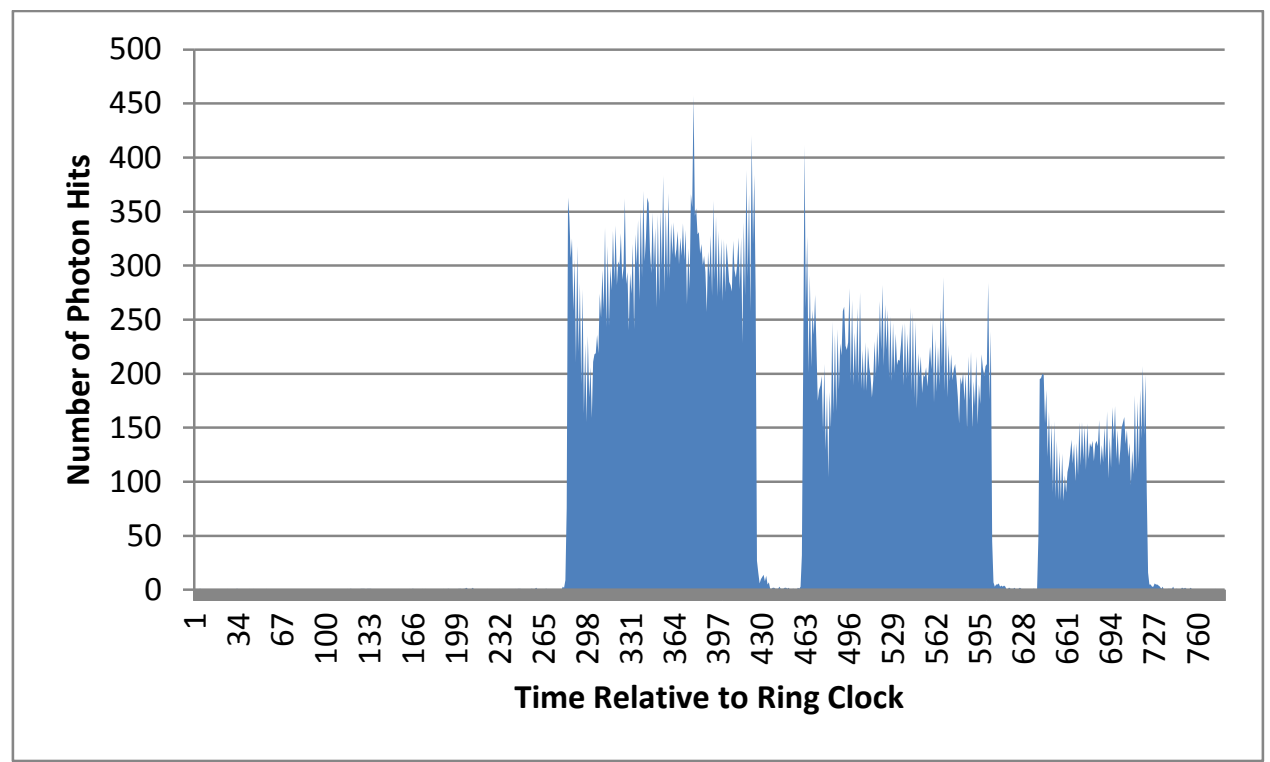

Figure 7: 3 Electron Bunch Train

Taken 8:20pm 07/25/2011

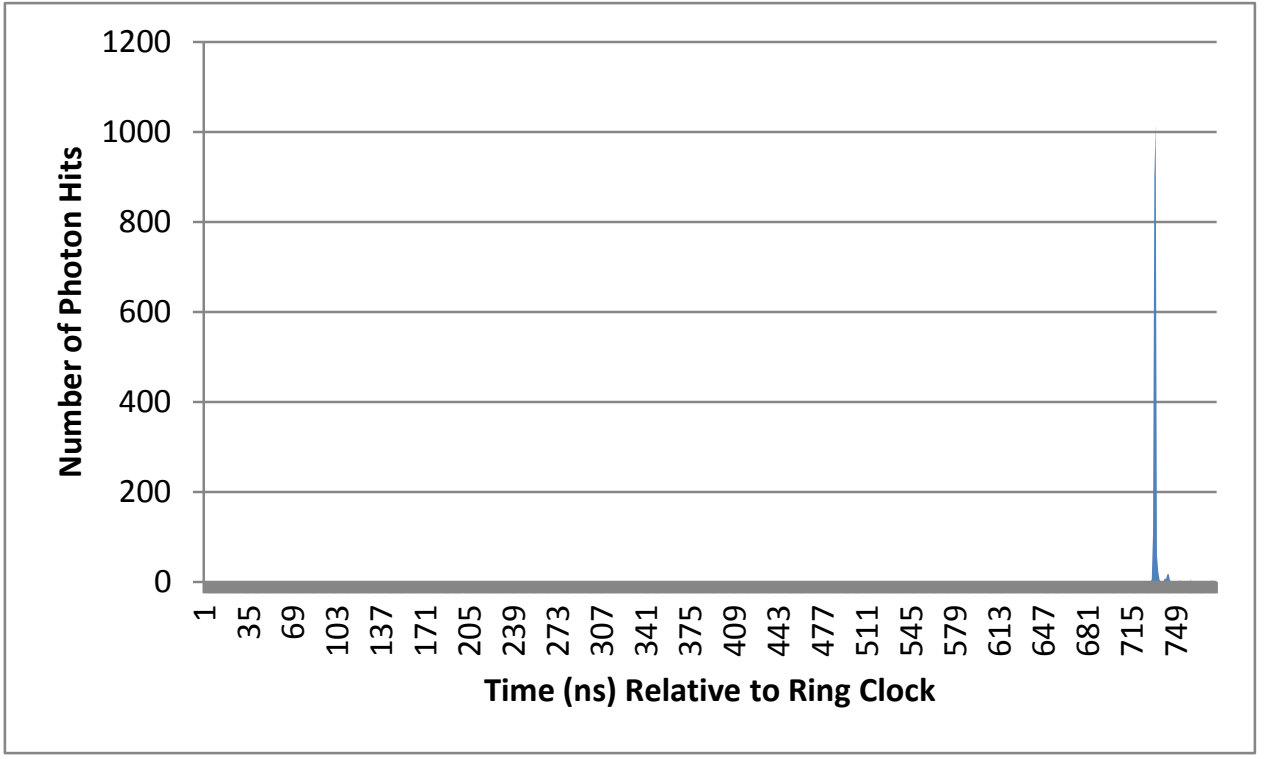

Figure 8: Single electron bunch at $5 \mathrm{~mA}$. Roughly $5 \%$ of charge is spilled into adjacent bunches. There is a small peak 6 buckets away, but we suspect that is the ringing effect from the PMT signal.

Taken: 5:52am 07/26/2011 


\section{Discussion/Conclusions}

The results of the single-photon counting measurements revealed much about the electron bunch distribution in SPEAR3. As seen in Figure 5, a short sampling time produces a roughly accurate electron distribution in the ring. However, when compared to Figure 6, the electron bunch pattern is much more accurate as the sampling time increases, as signal to noise ratio increases linearly to the square-root of $\mathrm{N}$ ( $\mathrm{N}$ being the number of samples).

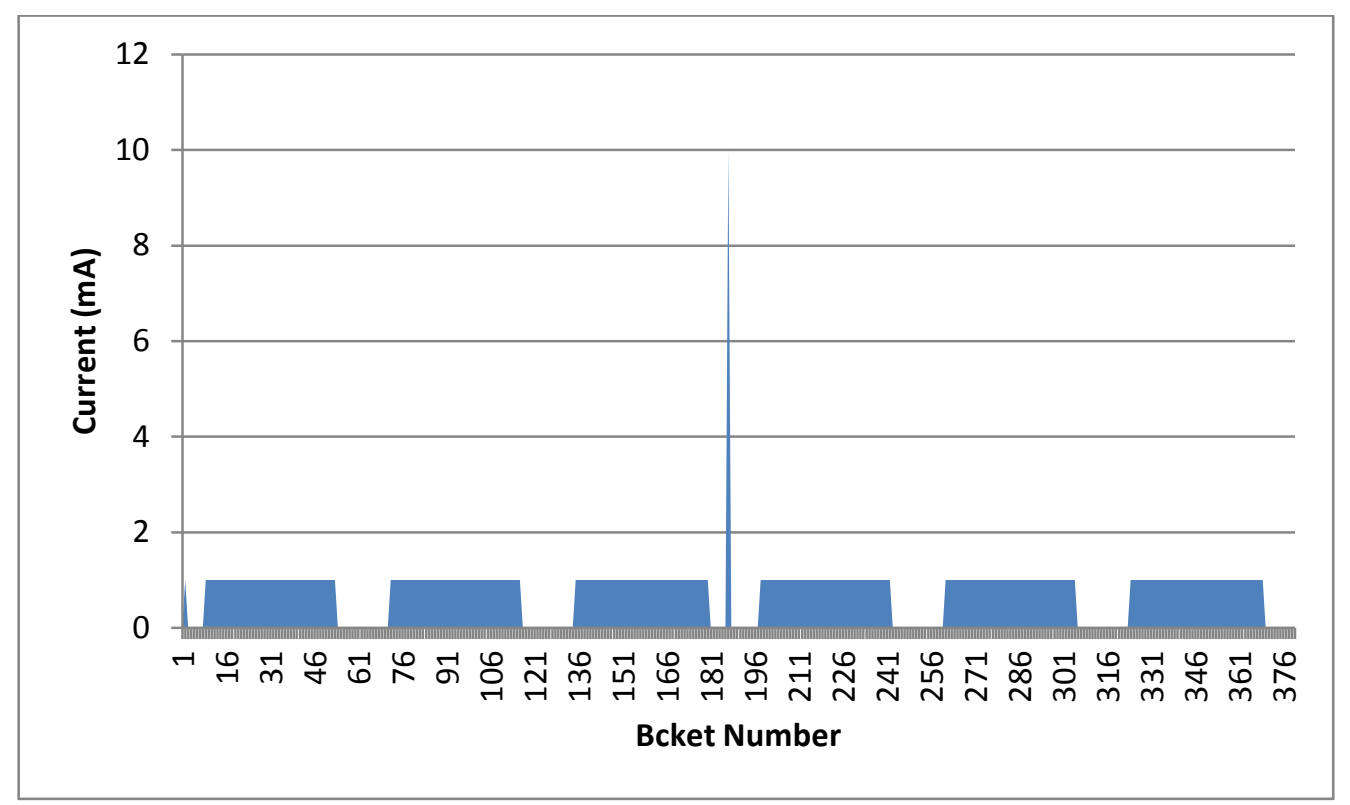

Figure 9: Ideal current distribution inside SSRL Storage ring.

Figure 9 represents the idea electron bunch distribution in the SSRL Storage at the time Figure 5 data is taken. While both distributions have roughly the same shape and timing, there are still many minor, yet important, differences.

The first noticeable difference is the unevenness of the bunch train in the experimental data. Noise, which in this case is ambient light and dark current from the PMT, contributes partially to the oscillation. However, the big spike at the beginning of each bunch train is more than just noise. The 
current injection system at SPEAR3 injects electron bunches into the assigned bucket without first measuring the current in that bucket. In other words, the system assumes what is left in the bucket and adds a predetermined amount of electrons into the bucket. If the remaining energy inside the bucket differs from assumption, then the total energy inside the bucket will differ from the target current after an injection. As seen in Figure 5, this "blind" injection resulted in more electrons at the beginning buckets of each train, as compared to the rest of the train. This result reveals a flaw in the top-off injection system at SSRL. The remaining current inside each bucket should be measured first, before injecting additional electrons. Such a feedback mechanism can help eliminate this problem.

Another important difference between the ideal and measured bunch patterns is evident in the "spilling" of electrons into adjacent buckets. Although electrons are injected into a bucket, oscillation and collision with other particles may knock an electron into an adjacent bucket. This result can be seen in the experimental results, as unfilled buckets near filled buckets have a small amount of current. This effect can be seen in Figure 8. For this specific bunch pattern, only one bucket is filled. However, in the results, observed electrons in the adjacent 8 buckets. Most of the charge was spilled into the 2 adjacent buckets, with roughly $5 \%$ of the Mundo bunch spilled in each bucket. This "spilling" effect is a known phenomenon, but the percentage of spilling must be monitored to ensure good beam quality.

\section{Acknowledgements}

Thanks to Jeff Corbett for being a wonderful mentor. He designed the experiment, supervised each step, and was of tremendous help to me. I worked in the same lab as Mitch Miller, and Jonathan Kamp. We collaborated on our projects and they helped me through many parts of my project. Dennis 
provided the counting card, which was essential to our experiment. Doug helped me with modifying the Lab View program. He is an experienced programmer and my program would not have worked if it wasn't for him. And thanks to the SULI program and DOE for this wonderful opportunity here at SLAC National Laboratory.

\section{References}

[1]Advanced Measurement Technology, Inc. “Ortec Model 9353100 Picosecond Time Digitizer, Hardware and Software User's Manual"

[2]Hamamatsu Inc. "Photon Counting Using Photomultiplier Tubes".

[3] Michael Wahl, PicoQuant GmbH. "Time-Correlated Single Photon Counting".

[4] A. Jeff* (CERN, Geneva, Switzerland \& University of Liverpool, UK), M. Andersen, S. Bozyigit,

E. Bravin, A. Boccardi, T. Lefevre, A. Rabiller, F. Roncarolo (CERN, Geneva, Switzerland);

C.P. Welsch (Cockcroft Institute, Daresbury, UK); A.S. Fisher (SLAC, Menlo Park, USA). "DESIGN FOR A LONGITUDINAL DENSITY MONITOR FOR THE LHC".

[5] M. D. Eisaman, J. Fan, A. Migdall, and S. V. Polyakov. "Single-photon sources and detectors" 